\title{
AVALIAÇÃO DA ATENÇÃO EM ADOLESCENTES ESCOLARES COM EXCESSO DE PESO ANTES E DEPOIS DO USO DE EXERGAMES EM GAMIFICAÇÃO: ENSAIO CLÍNICO RANDOMIZADO
}

\author{
EVALUATION OF ATTENTION IN SCHOOL ADOLESCENTS WITH \\ OVERWEIGHT BEFORE AND AFTER THE USE OF EXERGAMES IN \\ GAMIFICATION: RANDOMIZED CLINICAL TRIAL
}

\author{
Dimas Gabriel Sales Diniz ${ }^{1}$ \\ Carla Campos Muniz Medeiros ${ }^{2}$ \\ Danielle Franklin de Carvalho ${ }^{3}$
}

RESUMO: OBJETIVO: Avaliar os níveis de atenção sustentada e alternada de adolescentes escolares antes e depois do uso de exergames em gamificação. METODOLOGIA: Trata-se de uma pesquisa de ensaio clínico randomizado, na qual participaram 74 adolescentes, sendo 31 do grupo de intervenção e 43 do grupo controle. O grupo de intervenção foi submetido aos exergames em gamificação em um período de 8 semanas, 3 vezes por semana, durante 50 minutos, já o controle não recebeu nenhuma conduta. A randomização foi por escolas, devido à logística e ao viés de contaminação. Os adolescentes foram avaliados antes e depois do período de intervenção através do cálculo do IMC, para a seleção dos que estavam com sobrepeso ou obesidade, e do teste de trilhas, para observar os níveis de atenção dos que foram triados. A pesquisa aconteceu de março a julho de $2018 \mathrm{em}$ duas escolas municipais do ensino fundamental. RESULTADOS: A maioria dos indivíduos do grupo de intervenção eram do sexo feminino $(58,06 \%)$, já no controle eram do sexo masculino (53,49\%). A maioria dos indivíduos de ambos os grupos estavam em sobrepeso $(56,75 \%)$. A média etária do grupo intervenção foi de 12 anos, já no controle, de 13 anos. O grupo intervenção demonstrou melhora significante dos níveis de atenção (TTA: $p<0,001$ e TTB: $p<0,002$ ). CONCLUSÃO: Conclui-se que o uso de exergames em gamificação melhoram os níveis de atenção.

\footnotetext{
${ }^{1}$ Fisioterapeuta pela Universidade Estadual da Paraíba.

2 Doutora em Saúde da Criança e do Adolescente pela FCM-UNICAMP. Professora da Universidade Estadual da Paraíba.

3 Doutora em Saúde da Criança e do Adolescente pela Universidade Federal de Pernambuco. Professora da Universidade Estadual da Paraíba.
} 
Palavras chave: Atenção. Obesidade. Jogos de Vídeo. Saúde do adolescente. Sobrepeso.

ABSTRACT: OBJECTIVE: To evaluate the levels of sustained and alternated attention of school adolescents before and after the use of exergames in gamification. METHOD: This is a randomized clinical trial, in which 74 adolescents participated, 31 from the intervention group and 43 from the control group. The intervention group was submitted to exergames in gamification over a period of 8 weeks, 3 times a week, for 50 minutes, while the control did not receive any conduct. Randomization was carried out by schools, due to logistics and contamination bias. The adolescents were evaluated before and after the intervention period by calculating the BMI, to select those who were overweight or obese, and by the trail test, to observe the levels of attention of those who were screened. The research took place from March to July 2018 in two municipal elementary schools. RESULTS: Most of the individuals in the intervention group were female (58.06\%), while in the control group, they were male (53.49\%). Most individuals in both groups were overweight (56.75\%). The average age of the intervention group was 12 years old, while in control, 13 years old. The intervention group showed a significant improvement in attention levels (TTA: $p<0.001$ and TTB: $p$ <0.002). CONCLUSION: It is concluded that the use of exergames in gamification improves the levels of attention.

Keywords: Attention. Obesity. Video game. Adolescent health. Overweight. 


\section{INTRODUÇÃO}

Pode-se definir o sobrepeso/obesidade como uma condição clínica de ordem metabólica e/ou genética, na qual o indivíduo ingere mais energia do que gasta, gerando um desequilíbrio energético, e acumulando excesso de gordura em seu corpo, que fornece riscos para outras doenças (MORAES et al, 2014). Esta situação ocasiona um impacto negativo nos sistemas do organismo, dentre esses, o agravamento das funções cerebrais (FERREIRA et al, 2019; TEYCHENNE et al., 2015; ANTON-PADURARU et al, 2016).

A atenção é uma habilidade cerebral que é classificada como função executiva (FE) e pode ser definida como a capacidade que o indivíduo tem de perceber, selecionar e responder aos estímulos, através de uma complexa rede de operações mentais. Classifica-se em seletiva, sustentada e alternada. A atenção sustentada define-se por ser a competência em manter desempenho comportamental consistente em uma tarefa por um período prolongado de tempo, já a atenção alternada, é a aptidão de, durante a execução de uma tarefa, alternar o foco atencional entre mais de um estímulo (LURIA, 1979; SOHLBERG e MATEER, 1987; GOMES, 2010).

Nesse seguimento, o sobrepeso/obesidade demonstra relação significativa com o Transtorno de Déficit de Atenção e Hiperatividade (TDAH), a patologia psicológica que acomete a atenção e a mais frequente em crianças (PATTE et al, 2016; PEREZ- BONAVENTURA et al, 2015; JOHNSON et al, 2011). Acredita-se que a prevalência mundial de transtornos relacionados à atenção em crianças e adolescentes é cerca de 5,29\% (POLANCZYK et al, 2007).

Em relação ao excesso de peso, a "Organização Mundial da Saúde" estima que $6 \%$ da população infanto-juvenil do mundo tem sobrepeso/obesidade (WHO, 2017). A prevalência de tal patologia vem aumentando consideravelmente por todo mundo. Comparando-se proporção de obesos em todo o mundo, entre 1980 e 2014, observa-se que um aumento maior do que o dobro. Mudanças comportamentais, 
sobretudo devido à alimentação inadequada e ao sedentarismo, podem explicar esse aumento (WHO, 2015). No Brasil, as estimativas de prevalência de obesidade, segundo o Sistema de Vigilância de Fatores de Risco e Proteção para Doenças Crônicas por Inquérito Telefônico (VIGITEL), aumentaram de 15\% para 18\% de 2010 a 2014, em ambos os sexos (Ministério da Saúde, 2018). Assim, ao perceber o crescente aumento na prevalência do sobrepeso e da obesidade, os níveis de atenção em crianças e adolescentes se tornam algo com o que se preocupar, visto que sofrem impacto negativo do excesso de peso (CORTESE e TESSARI, 2017; WHO, 2015; Ministério da Saúde, 2018).

Apesar disso, também é compreendido que há uma correlação oposta, na qual níveis baixos de atenção podem promover risco de estar em excesso de peso. Observa-se que indivíduos com TDAH têm pelo menos 1,23 vezes mais probabilidade de se tornarem obesos do que aqueles sem TDAH (AGUIRRE CASTANEDA et al, 2016; GUNGOR et al, 2016; KUMMER et al, 2016), logo, compreende-se que um tratamento para um fator pode afetar o outro de maneira positiva, especialmente se for utilizado um tratamento baseado em atividade física regular, o qual já é comprovado que gera ótimos efeitos no corpo (MEHREN et al, 2020; VILLA-GONZÁLEZ et al, 2020; ZANG, 2019), além disso, quando se entende a relação do TDAH nos processos neurobiológicos da desregulação dopaminérgica, percebe-se que o efeito da prática de exercícios é importante no equilíbrio hormonal, haja vista a necessidade de controlar os circuitos de reforço, gratificação e prazer, afetados em indivíduos com TDAH (VELASCO et al, 2015).

Portanto, sabendo que a adolescência é um período fundamental, já que frequentemente é quando há um baixo nível de atividade física, formação do perfil neurocognitivo, estabelecimento de aspectos comportamentais e desenvolvimento na composição corporal, torna-se efetivo implementar medidas de prevenção e intervenção nesse momento importante (FERREIRA et al, 2019; ECHEVERRIA et al, 2014 ; KATZMARZYK et al., 2014).

Nesse sentido, ferramentas lúdicas que tornem a prática de atividade física um momento divertido e prazeroso, incentivando as mesmas, são sempre bem-vindas, por isso meios tecnológicos vem se tornando um instrumento que traz grandes benefícios à saúde. Dentro dessa perspectiva, a modalidade de jogos de realidade 
aumentada denominada Exergames tem sido estudada e aprovada na proposição de estímulo para o exercício físico, através do qual o indivíduo utiliza os movimentos do corpo para controlar o jogo, viabilizando um maior consumo de energia por parte dos jogadores (RODRIGUES et al, 2013; WILLIAMS e AYRES, 2020; CEBOLLA et al, 2015; LEE, 2017), sendo assim, os efeitos que atividades físicas comuns têm, também podem ser obtidos com o uso de jogos, portanto, considerando o exposto, este estudo objetivou avaliar os níveis de atenção sustentada e alternada em adolescentes escolares com excesso de peso antes e depois da utilização de exergames gamificados durante 50 minutos e 3 vezes por semana.

\section{METODOLOGIA}

Trata-se de um ensaio clínico randomizado controlado com dois grupos de comparação: o experimental, no qual os participantes foram submetidos à prática do exercício físico com auxílio do exergame associada à gamificação, em um período de 8 semanas, 3 vezes por semana, durante 50 minutos; e o controle, no qual não houve intervenção, sendo utilizado apenas para comparação de dados.

A randomização da intervenção foi realizada por escolas e não por indivíduos, devido a logística e ao viés de contaminação.

O estudo ocorreu no período de março a julho de 2018, em duas escolas públicas de ensino fundamental e envolveu 74 adolescentes com sobrepeso ou obesidade distribuídos da seguinte forma: 31 no grupo de intervenção e 43 no controle.

$\mathrm{Na}$ zona urbana do município de Campina Grande, PB, existem 20 escolas municipais, de ensino fundamental II, que atendem alunos do $5^{\circ}$ ao $9^{\circ}$ ano, nos turnos da manhã e/ou tarde. Para escolha do local do estudo foi realizado um levantamento do número de alunos matriculados nessas escolas e, posteriormente, distribuição desses números em quartis. Foram selecionados para o sorteio as escolas que apresentaram quantidade de alunos acima do terceiro quartil (298 
alunos). Entre as cinco escolas que se encontravam acima do terceiro quartil foram sorteadas duas escolas, 1 para cada grupo.

Todos adolescentes pertencentes as escolas selecionadas foram convidados a participarem do estudo desde que estivessem dentro dos critérios de elegibilidade. Foram incluídos no estudo adolescentes com sobrepeso/obesidade, entre 10-16 anos e cursando do $5^{\circ}$ ao $9^{\circ}$ ano do ensino fundamental, e excluídos aqueles que apresentassem uma das seguintes condições: estar na vigência de algum tratamento para emagrecer, gravidez ou amamentação, síndrome genética, situações que não permitissem a realização de atividade física, como limitação motora ou mental, ou doenças cuja a realização de atividade física pudessem exacerbá-las.

No início da pesquisa, foi realizada, pelos pesquisadores, uma visita nas escolas selecionadas para esclarecimento aos gestores sobre os detalhes do estudo. Posteriormente, foi realizado o treinamento da equipe de pesquisa, visando a padronização dos procedimentos que foram utilizados na coleta de dados.

Realizou-se, nas escolas selecionadas, uma triagem do estado nutricional para a identificação dos alunos com sobrepeso ou obesidade. Os adolescentes elegíveis foram convidados a participarem da pesquisa e, após explicação do estudo pelos pesquisadores, os alunos que tiveram interesse em participar assinaram 0 Termo de Assentimento e seus responsáveis, o Termo de Consentimento Livre Esclarecido.

O estudo constou de três fases: a primeira, antes da intervenção, na qual foi realizada a avaliação antropométrica e aplicados os instrumentos para avaliação da atenção; a segunda que foi a intervenção e a terceira que constou da reavaliação dos dados iniciais.

Para classificação do estado nutricional foi realizada a antropometria. A mensuração da altura e peso foram obtidos em duplicata e foi considerada a média dos valores das duas aferições. Para aferição da altura, foi utilizado um estadiômetro portátil, marca Avanutri® com precisão de $0,1 \mathrm{~cm}$ e para a identificação do peso foi utilizada uma balança digital Tanita® cuja capacidade é de $150 \mathrm{~kg}$ e precisão de 0,1 kg. Para obtenção da medida o adolescente tinha de estar com roupas leves, sem calçados e adereços. A diferença entre as medidas de estatura deveria ser igual ou 
inferior a $0,5 \mathrm{~cm}$. A diferença entre as medidas de peso deveria ser igual ou inferior a 100 gramas.

A atenção alternada e sustentada foi avaliada através dos seguintes instrumentos: cronômetro digital portátil Moure Jar® - mj1822 - "Zona Livre" e "Teste de Trilhas", numa sala fechada, silenciosa, sem interferências exteriores, antes e depois da intervenção.

O "Teste de Trilhas" compôs-se de duas partes, ambas consistindo em 25 círculos distribuídos por uma folha de papel. Na Parte $A$, os círculos foram numerados de 1 a 25 e o paciente desenhou linhas para conectar os números em ordem crescente. Na Parte $B$, os círculos incluíam números $(1-13)$ e letras $(A-L)$; como na Parte A, o paciente desenhou linhas para conectar os círculos em um padrão ascendente, mas com a tarefa adicional de alternar entre os números e letras (ou seja, 1-A-2-B-3-C, etc.). O paciente foi instruído a conectar os círculos o mais rápido possível, sem levantar a caneta ou o lápis do papel. Mediu-se o tempo que o paciente levou para conectar a "trilha" com um cronômetro. Se o paciente cometesse um erro, indicava-se imediatamente e permitia-se que o paciente corrigisse. Os erros afetavam a pontuação do paciente apenas porque a correção de erros era incluída no tempo de conclusão da tarefa. Não era necessário continuar o teste se o paciente não tivesse completado as duas partes após cinco minutos.

A intervenção constou da realização de atividade física, durante 50 minutos, três vezes na semana e durante oito semanas. Com essa finalidade, foi utilizada a plataforma "XBOX 360" juntamente com o acessório "Kinect" (Microsoft@) para possibilitar ao usuário o controle e interação apenas com comando dos movimentos corporais, fazendo com que ele realizasse atividade física. O jogo de realidade alternada selecionado foi o "Just Dance" versões 2014, 2015, 2016, 2017 e 2018.

As atividades foram realizadas em salas reservadas na escola selecionada, em grupos de quatro participantes, sendo supervisionadas e controladas através do registro da presença dos adolescentes no dia da atividade. Utilizou-se um frequencímetro MULTILASER® Atrio antes (para cálculo da frequência de treino) e durante (para monitorização da intensidade do exercício) a atividade.

As danças utilizadas para intervenção foram previamente selecionadas, sendo incluídas aquelas que podiam levar a uma intensidade moderada, e reunidas 
em bloco de 10 (GBLOCK). Para escolha dos jogos, foi utilizado o "Just Dance" 2014 até o 2018. Essa seleção foi realizada por alunos de educação física com experiência na utilização dessa tecnologia para promoção de atividade física.

Para aumentar o engajamento dos adolescentes na atividade de intervenção, realizou- se uma estratégia de gamificação através da elaboração de blocos com 10 músicas do "Just Dance", com a finalidade de haver realização da intervenção semanalmente e de premiações para a equipe e o adolescente que atingissem a maior pontuação, causando uma situação de cooperação e competição.

Esse estudo fez parte de uma pesquisa maior intitulada de "Impacto do exergame na aptidão cardiorrespiratória, macro e microcirculação de adolescentes com excesso de peso: estudo de intervenção randomizado", aprovada pelo comitê de ética em Pesquisa da Universidade Estadual da Paraíba sob o CAAE:840195183.0000.5187 e registrada no Clinical Trials (NCT03532659).

A análise estatística foi realizada no "Statistical Package for the Social Sciences" (SPSS), versão 22.0. Os dados foram digitados em um banco feito no "Microsoft Excel 2016" e, posteriormente, foram repassados para o SPSS. Após isso, analisou-se a distribuição da população por idade e sexo. Para comparação da distribuição das características sociodemográficas e do estado nutricional, entre o grupo intervenção e controle, foi realizado o teste do qui-quadrado. Para avaliação da atenção seletiva e alternada foi utilizado o teste Wilcoxon, uma vez que apresentou distribuição assimétrica no teste de Kolmogorov-Sminorv. Para todas as análises foi adotado um nível de significância de $5 \%$.

\section{RESULTADOS E DISCUSSÃO}

Houve uma população de 74 indivíduos, sendo 41,89\% (31) do grupo de intervenção e 58,10\% (43) do grupo controle.

O grupo de intervenção foi composto por adolescentes com uma mediana de idade de 12 anos, sendo 58,06\% (18) do sexo feminino e 41,93\% (13) do sexo masculino, no qual $61,29 \%$ (19) achou-se em sobrepeso e $38,71 \%$ (12) em 
obesidade, já o grupo controle formou-se por indivíduos com mediana de idade de 13 anos, sendo 53,49\% (23) do sexo masculino e 46,51\% (20) do sexo feminino, no qual $53,48 \%$ (23) em sobrepeso e $46,62 \%$ em obesidade (TABELA 1).

Tabela 1. Comparação da distribuição dos sexos, idades, estados emocionais e estados nutricionais de ambas as escolas, Campina Grande, PB, 2018.

\begin{tabular}{lccccc}
\hline INTERVENÇÃO & \multicolumn{5}{c}{ CONTROLE } \\
\cline { 1 - 4 } & MEDIANA & IQ & MEDIANA & IQ & P \\
\hline IDADE & 12 & 2 & 13 & 2 & 0,007 \\
\hline SEXO & & & \multirow{2}{*}{0,327} \\
\hline Masculino & $13(41,93 \%)$ & $23(53,49 \%)$ & \\
\hline Feminino & $18(58,06 \%)$ & $20(46,51 \%)$ & \multirow{2}{*}{0,504} \\
\hline ESTADO NUTRICIONAL & $19(61,29 \%)$ & $23(53,48 \%)$ & \\
\hline \multicolumn{2}{l}{ Obobrepesidade } & $12(38,71 \%)$ & $20(46,62 \%)$ & \\
\hline
\end{tabular}

p: nível de significância estatística; IQ: Intervalo Interquartil.

Para a avaliação da atenção sustentada e alternada, as duas partes do "Teste de Trilhas" foram analisadas separadamente (TTA e TTB). Observou-se então que houve significância nos resultados após o período de intervenção em ambas as escolas. Analisando as medianas e os intervalos interquartis, viu-se que em ambos os grupos houve diminuição do tempo de teste, indicando uma melhora na atenção nas duas populações (TABELA 2).

Tabela 2. Comparação dos níveis de atenção sustentada e alternada através do "Teste de Trilhas", Campina Grande, PB, 2018.

\begin{tabular}{|c|c|c|c|c|c|c|c|c|c|c|}
\hline & \multicolumn{5}{|c|}{ INTERVENÇÃO } & \multicolumn{5}{|c|}{ CONTROLE } \\
\hline & \multicolumn{2}{|c|}{ Inicial } & \multicolumn{2}{|c|}{ Final } & \multirow[t]{2}{*}{$\mathbf{P}$} & \multicolumn{2}{|c|}{ IniciaL } & \multicolumn{2}{|c|}{ Final } & $\mathbf{P}$ \\
\hline & Md & $\mathrm{IQ}$ & Md & IQ & & Md & $\mathrm{IQ}$ & Md & IQ & \\
\hline TTA & 58,38 & 20,03 & 44,90 & 24,19 & 0,001 & 49,22 & 21,27 & 40,97 & 20,87 & 0,001 \\
\hline TTB & 137,87 & 56,94 & 97,88 & 38,81 & 0,002 & 118,47 & 101,22 & 84,6 & 52,68 & 0,001 \\
\hline
\end{tabular}


Para explicar a situação e devido a distribuição da variável, calculou-se a variação dessa variável em cada escola, fazendo-se a comparação das variações entre as escolas através do teste $U$ de Mann-Whitney (TABELA 3). Como resultado, houve significância da variação apenas na primeira parte do teste (TTA), além disso, notou-se que a variação foi maior no grupo de intervenção do que no grupo controle.

Tabela 3. Comparação das variações dos níveis de atenção sustentada e alternada através do "Teste de Trilhas", Campina Grande, PB, 2018.

\section{Intervenção}

Controle

\begin{tabular}{cccccc}
\hline TTA & Mediana & Intervalo Interquartil & Mediana & Intervalo Interquartil P (da variação) \\
& 15,33 & 19,97 & 8,4 & 17,72 & 0,029 \\
TTB & 37,35 & 53,68 & 29,53 & 52,34 & 0,507 \\
\hline
\end{tabular}
p: nível de significância estatística; TTA: Teste de Trilhas - Parte A; TTB: Teste de Trilhas - Parte B.

Logo, entende-se que através do "Teste de Trilhas", observou-se uma melhora significante na atenção sustentada e alternada entre os indivíduos que foram submetidos à intervenção e, apesar dos participantes da escola controle também terem demonstrado uma diminuição no tempo do teste, não foi tão expressiva quanto na escola intervenção. Provavelmente a escola controle desenvolveu atividades que favoreceram um pouco a atenção sustentada e alternada do grupo controle, visto que, na avaliação inicial, os participantes do grupo controle já demonstravam menor tempo de teste quando comparados ao grupo intervenção.

Tais resultados sobre os níveis de atenção corroboram com o que alguns estudos já vem comprovando: que a utilização de videogames em pacientes com níveis diminuídos de atenção é benéfica, melhorando a qualidade de vida dos indivíduos (HEINRICH et al, 2007; LAWRENCE et al, 2002; BAVELIER et al; 2011).

Em relação aos resultados colhidos referentes a atenção sustentada e alternada, a escola controle provavelmente sofreu alteração significante devido a atividades promovidas pela escola, como jogos de mesa. Segundo Guerrelhas et al (2002), jogos de mesa têm efeito positivo nos níveis de atenção. Tintori et al (2011) afirma que os jogos de mesa podem ser considerados um método de tratamento 
para indivíduos com diagnóstico de TDAH, logo, compreendendo o exposto, não apenas justifica-se os resultados, como também se sugere que o uso de exergames possui mais efetividade do que jogos de mesa na melhora dos níveis de atenção, todavia, são necessários estudos mais controlados de comparação de ambos os fatores, a fim de se afirmar com certeza tais conclusões.

Por fim, uma observação interessante a ser feita é que, segundo Cypel (2007), quanto maior a faixa etária, maiores serão os níveis de atenção e, tendo em vista que a escola controle possui uma mediana de idade maior que a escola intervenção, compreende-se que tinham certa vantagem, no entanto, é surpreendente ver quão efetiva foi a proposta de intervenção, afinal os indivíduos com menor faixa etária e, conseguintemente, menores níveis de atenção, foram os que demonstraram maiores diferenças positivas nas avaliações finais.

\section{CONCLUSÃO}

O exergame ou videogame ativo (VGA) é uma ferramenta lúdica que utiliza o ambiente de realidade virtual aumentada para proporcionar diversão. Para participar dos jogos, é necessário o uso do próprio corpo, a fim de se controlar o personagem e interagir no ambiente. Assim como em outros tipos de jogos, a gamificação, estratégia de jogos cujo o objetivo é cativar os participantes através de desafios e premiações, pode ser usada nos exergames.

Sendo assim, observou-se no presente estudo, que o uso de exergames gamificados têm efeito positivo significante nos níveis de atenção sustentada e alternada em adolescentes com excesso de peso. Tal compreensão auxilia na seleção de técnicas para o tratamento de patologias que afetam a atenção, colaborando também com a quebra do preconceito com o uso de novas tecnologias, além disso, motiva a inserção do fisioterapeuta, profissional que se utiliza da prescrição de exercícios e de tecnologias, como formas de terapia física (gameterapia), para o tratamento de doenças de origem mental, visto que o âmbito mental pode ser modulado pelo físico. 
Ainda assim, sugere-se que mais pesquisas na área com amostras maiores e mais controladas sejam feitas, com a finalidade de saber se o exergame, ao melhorar os níveis de atenção, consegue ajudar no processo de perda de peso, visto que o excesso de peso está bastante relacionado com os níveis baixos de atenção.

\section{REFERÊNCIAS BIBLIOGRÁFICAS}

AGUIRRE CASTANEDA, R. L.; KUMAR, S.; VOIGT, R.G.; et al. Childhood attentiondeficit/hyperactivity disorder, sex, and obesity: a longitudinal population-based study. Mayo Clinic Proceedings, v. 91, p. 352-361, 2016.

ANTON-PADURARU, D.T; TESLARIU, O.; MOCANU, V. Influence of Sleep on Obesity in Children. Revista Medico-Chirurgicala a Societatii de Medici si Naturalisti din lasi, lasi, Romênia, v. 120, n. 2, p. 239-243, 2016.

BAVELIER, D.; GREEN, C. S.; HAN, D. H., et al. Brains on video games. Nature Reviews Neuroscience, v. 12, p. 763-768, 2011.

CEBOLLA, A.; ÁlVAREZ-PITTI, J. C.; PROVINCIALE, J. G. et al. Alternative options for prescribing physical activity among obese children and adolescents: brisk walking supported by an exergaming platform. Nutricion Hospitalaria, v. 31, p. 841-848, 2015.

CORTESE, S.; TESSARI, L. Attention-Deficit/Hyperactivity Disorder (ADHD) and Obesity: Update 2016. Current Psychiatry Reports, v. 19, n. 4, p. 1-15, 2017.

FERREIRA, P. D. S.; SZWARCWALD, C. L.; DAMACENA, G. N. Prevalence of obesity and associated factors in the Brazilian population: a study of data from the 2013 National Health Survey. Revista Brasileira de Epidemiologia, v. 22, p. 1-14, 2019.

GOMES, J. O. Teste de Atenção Dividida Alternada. Psico-USF, v. 15, n. 3, p. 419-420, set./dez de 2010.

GUERRELHAS, F.; BUENO, M.; SILVARES, E.F.M. Grupo de ludoterapia comportamental X Grupo de espera recreativo infantil. Revista Brasileira de Terapia Comportamental e Cognitiva, v. 2, n. 2, p. 157-169, 2002.

GUNGOR, S.; CELILOGLU, O. S.; RAIF, S. G.; et al. Malnutrition and obesity in children with ADHD. Journal of Attention Disorders, v. 20, p. 647-652, 2016.

HEINRICH, H.; GEVENSLEBEN, H.; STREHL, U. Annotation: Neurofeedback - train your brain to train behavior. Journal of Child Psychology and Psychiatry, v. 48, p. 3-16, 2007.

JOHNSON, R.J.; GOLD, M.S., JOHNSON, D.R., et al. Attention-deficit/hyperactivity disorder: is it time to reappraise the role of sugar consumption? Postgraduate Medicine, v. 123, p. 39-49, 2011.

KATZMARZYK, P. T.; BARLOW, S.; BOUCHARD, C.; et al. An evolving scientific basis for the prevention and treatment of pediatric obesity. International Journal of Obesity, v. 38, n. 7, p. 887-905, 2014. 
KUMMER, A.; BARBOSA, I. G.; RODRIGUES, D. H.; et al. Frequency of overweight and obesity in children and adolescents with autism and attention deficit/hyperactivity disorder. Revista Paulista de Pediatria: Orgao Oficial da Sociedade de Pediatria de Sao Paulo, v. 34, p. 7177, 2016.

LAWRENCE, V.; HOUGHTON, S.; TANNOCK, R., et al. ADHD outside the laboratory: Boys' executive function performance on tasks in videogame play and on a visit to the zoo. Journal of Abnormal Child Psychology, v. 30, p. 447-462, 2002.

LEE, S.; KIM, W.; PARK, T. et al. The psychological effects of playing exergames: a systematic review. Cyberpsychology, Behavior, and Social Networking, v. 20, p. 513-532, 2017.

LURIA, A. Curso de Psicologia geral. Rio de Janeiro: Civilização Brasileira, 1979 (4 vols.).

MEHREN, A.; REICHERT, M.; COGHILL, D. et al. Physical exercise in attention deficit hyperactivity disorder - evidence and implications for the treatment of borderline personality disorder. BioMed Central, v. 7, n. 1, p. 1-11, 2020.

Ministério da Saúde. Vigitel Brasil 2017 - Vigilância de fatores de risco e proteção para doenças crônicas por inquérito telefônico. Brasília: Ministério da Saúde, Agência Nacional de Saúde Suplementar; 2018.

MORAES, J. M.; CAREGNATO, R. C. A.; SCHNEIDER, D. S. Qualidade de vida antes e após a cirurgia bariátrica. Acta Paulista de Enfermagem, São Paulo, v.27, n.2, p. 157-164, 2014.

PEREZ-BONAVENTURA, I.; GRANERO, R.; EZPELETA, L. The relationship between weight status and emotional and behavioral problems in Spanish preschool children. Journal Pediatric Psychology, v. 40, p. 455-463, 2015.

POLANCZYK, G.; DE LIMA, M.S.; HORTA, B.L., et al. The worldwide prevalence of ADHD: a systematic review and metaregression analysis. American Journal of Psychiatry, v. 164, p. 942-948, 2007.

RODRIGUES, C. E. M. Importância do Lúdico no Impacto Psicológico da Hospitalização Infantil. Biblioteca Digital da Universidade Jean Piaget de Cabo Verde, 2013.

SOHLBERG, M. M.; MATEER, C. A. Effectiveness of an attention-training program. Journal of Clinical and Experimental Neuropsychology, v. 9, n. 2, p. 117-130, 1987.

TEYCHENNE, Megan. et al. The association between sedentary behaviour and risk of anxiety: a systematic review. BMC Public Health, v. 15, p. 513, 2015.

TINTORI, F.; BAST, D.F.; PITTA, M.R. Jogo na terapia comportamental em grupo de crianças com TDAH. Acta Comportamentalia, v. 19, n. 2, p. 225-239, 2011.

VELASCO, R. M.; BARBUDO, E.; PÉREZ-TEMPLADO, J., et al. Review of the Association Between Obesity and ADHD. Actas Españolas de Psiquiatría, v. 43, n. 1, p. 16- 23, 2015.

VILLA-GONZÁLES, R.; VILLALBA-HEREDIA, L.; CRESPO, I. et al. A systematic review of acute exercise as a coadjuvant treatment of ADHD in young people. Psicothema, v. 32, n. 1 , p. $67-74,2020$.

World Health Organization. Global status report on noncommunicable diseases 2014. Genebra: World Health Organization; 2015.

WHO. World Health Organization. Guideline: assessing and managing children at primary health-care facilities to prevent overweight and obesity in the context of the double burden of malnutrition. Updates for the Integrated Management of Childhood Illness (IMCl). WHO. 
Avaliação da Atenção em Adolescentes Escolares com Excesso de Peso Antes e Depois do Uso de Exergames em Gamificação: Ensaio Clínico Randomizado

Geneva, Suíça. $\quad$ p.2017. $\quad$ Disponível em:

<http://apps.who.int/iris/bitstream/10665/259133/1/9789241550123-eng.pdf?ua=1>.

WILLIAMS, W. M.; AYRES, C. G. Can Active Video Games Improve Physical Activity in Adolescents? A Review of RCT. International Journal of Environmental Research and Public Health, v. 17, n. 2, p. 1-10, 2020.

ZANG, Y. Impact of physical exercise on children with attention deficit hyperactivity disorders Evidence through a meta-analysis. Medicine, v. 48, n. 96, p. 1-10, 2019. 